\section{THU0092 DEFINING A LEARNING CURVE FOR CLINICIANS PERFORMING ULTRASOUND-GUIDED DEFINING A LEARNING CURVE FOR CLINICIANS PERFORMING ULTRASOUND-GUIDED NEEDLE SYNOVIAL BIOPSIES: A RETROSPECTIVE ANALYSIS OF THE EXPERIENCE AT THE EXPERIMENTAL MEDICINE AND RHEUMATOLOGY, QUEEN MARY UNIVERSITY OF LONDON}

Alessandra Nerviani ${ }^{1}$, Frances Humby ${ }^{1}$, Gloria Lliso Ribera ${ }^{1}$, Felice Rivellese ${ }^{1}$, Stephen Kelly ${ }^{2}$, Rebecca Hands ${ }^{1}$, Costantino Pitzalis ${ }^{1} .{ }^{1}$ William Harvey Research Institute, Queen Mary University of London, United Kingdom, Centre for Experimental Medicine and Rheumatology, London, United Kingdom; ${ }^{2}$ Barts Health NHS Trust, Rheumatology, London, United Kingdom

Background: Despite the significantly better outcome reached by patients with Rheumatoid Arthritis following the introduction of the biologics, the absence of predictors of individual response to the available agents constitutes a huge unmet clinical need. The histological analysis of the diseased synovial tissue (ST) may represent a valuable prognostic tool. The ultrasound-guided needle synovial biopsy (US-nSB) is a safe, effective and increasingly used method to retrieve ST from both large and small joints. Therefore, assessing the 'learning curve' for clinicians training in this technique is critically important.

Objectives: To retrospectively evaluate the learning curve for clinicians performing US-nSB at the Centre for Experimental Medicine and Rheumatology, Queen Mary University of London.

Methods: The performance of 5 clinicians has been evaluated from their first to $>20$ procedures based on the training phase (Table). The standard teaching method included: US/US-guided injections; observation of US-nSB; initial procedures occurring under the strict active supervision of an expert.

\begin{tabular}{|c|c|c|c|c|c|c|}
\hline Training Phase & $\begin{array}{l}1^{\text {st }}- \\
10^{\text {th }}\end{array}$ & $\begin{array}{l}11^{\text {th }}- \\
20^{\text {th }}\end{array}$ & $\begin{array}{l}21^{\text {st. }} \\
30^{\text {th }}\end{array}$ & $\begin{array}{l}31^{\text {st }}- \\
40^{\text {th }}\end{array}$ & $\begin{array}{l}41^{\text {st. }} \\
50^{\text {th }}\end{array}$ & $>50^{\text {th }}$ \\
\hline $\begin{array}{l}\text { Total } n^{\circ} \text { of procedures } \\
\text { included }\end{array}$ & 50 & 50 & 43 & 35 & 30 & 90 \\
\hline
\end{tabular}

Results: The total number of ST fragments retrieved during the procedure did not significantly change during the learning phase $(17.3 \pm 3.5$ from $1^{\text {st }}$ to $10^{\text {th }}$ biopsy vs $17.2 \pm 3.4$ if expert performer). Independently of the training phase, a significantly higher number of samples were retrieved from knees/wrists in comparison with metacarpo-, metatarso-, and proximal inter-phalangeal joints. The average weight of a ST fragment at the beginning of the learning curve was comparable with the mean weight of ST samples retrieved by expert performers $(3.7 \pm 2.5 \mathrm{mg}$ from $1^{\text {st }}$ to $10^{\text {th }}$ biopsy vs $2.9 \pm 2.4 \mathrm{mg}$ if experts). ST from knees had a significantly higher mean weight, which could be explained by the more frequent use of a $14 \mathrm{G}$ (rather than 16G) needle. At the beginning of the learning curve, $48.2 \% \pm 29.5 \%$ of the retrieved ST-samples were considered histologically 'gradable' (visible lining and/or clear sub-lining based on H\&E/CD68 IHC staining); the rate of gradable ST progressively increased and remained $\approx 60 \%$ when $>20$ procedures have been performed. The duration of the US-nSB significantly decreased during the learning time $\left(49 \pm 18 \mathrm{~min}\right.$ from $1^{\text {st }}$ to $10^{\text {th }}$ biopsy vs $30 \pm 9 \mathrm{~min}$ if expert performer). No serious adverse events (AEs) such as haemarthrosis or joint infection have occurred during the learning time. The overall rate of minor AEs (e.g., minor bleeding) was not the highest at the beginning of the training. During the $11^{\mathrm{st}}-10^{\text {th }}$ procedures, $18.2 \%$ of the patients reported severe pain compared to the average $8.4 \%$.

Conclusion: In this single-centre retrospective study we showed that, with an appropriate training and the presence of the initial strict supervision, performing US-nSB is safe and effective since the beginning of the learning curve. Further prospective and multi-centre studies are needed to confirm the optimal learning time and training method.

\section{REFERENCES:}

[1] Ultrasound-guided synovial biopsy: a safe, well-tolerated and reliable technique for obtaining high-quality synovial tissue from both large and small joints in early arthritis patients. S Kelly et al. Ann Rheum Dis. 2015 Mar;74 (3):611-7.

[2] Patient-reported outcomes and safety in patients undergoing synovial biopsy: comparison of ultrasound-guided needle biopsy, ultrasound-guided portal and forceps and arthroscopic-guided synovial biopsy techniques in five centres across Europe. SA Just, et al. RMD Open. 2018 Oct 26;4(2): e000799.
Disclosure of Interests: Alessandra Nerviani: None declared, Frances Humby: None declared, Gloria Lliso Ribera: None declared, Felice Rivellese: None declared, Stephen Kelly: None declared, Rebecca Hands: None declared, Costantino Pitzalis Grant/research support from: Celgene DOI: 10.1136/annrheumdis-2019-eular.7302

\section{THU0093 BASAL METABOLIC RATE AND CHARLSON COMORBIDITY INDEX ARE INDEPENDENT PREDICTORS OF METABOLIC SYNDROME IN PATIENTS WITH RHEUMATOID ARTHRITIS}

Dong-Jin Park, Haimuzi Xu, Sung-Eun Choi, Ji-Hyoun Kang, Shin-Seok Lee. Cheonnam National University Hospital, Gwangju, Korea, Rep. of (South Korea)

Background: Metabolic syndrome (MetS) is associated with 2-fold increase in cardiovascular disease (CVD) and 1.5-fold increase in allcause mortality in the general population. The overall pooled prevalence of MetS in the meta-analysis of rheumatoid arthritis (RA) patients is $30.65 \%$. Along with high prevalence of MetS in RA, several studies showed the contribution of MetS to the increased risk of CVD or cardiovascular mortality in RA patients. Although controversial, the inflammatory activity of RA was significantly associated with MetS in several cross-sectional studies. However, no prospective studies have confirmed an increased risk of MetS development during the course of RA.

Objectives: This 2-year prospective study investigated the predictors of metabolic syndrome (MetS) in patients with rheumatoid arthritis (RA).

Methods: We recruited 319 consecutive RA patients who did not have MetS at baseline. MetS was defined according to the modified NECP/ ATP III 2005 for Asian populations. Predictors of MetS were assessed using univariate and multivariate logistic regression analyses.

Results: Of the 247 RA patients who finished 2-year follow-up, 37 $(15.0 \%)$ developed MetS. At baseline, the patients who developed MetS were older and had higher BMI $(P=0.002)$, higher waist circumference $(P=0.011)$, higher waist-hip ratio $(P=0.035)$, greater skeletal muscle mass $(P=0.005)$, higher body fat mass $(P=0.011)$, higher percent body fat $(P=0.004)$, lower basal metabolic rate $(P=0.004)$ and higher Charlson comorbidity index score $(P<0.001)$. The percent of diabetes $(P=0.007)$ and hypertension $(P<0.001)$ were higher in patients with MetS than without MetS. And they also had elevated glucose $(P=$ 0.004), triglyceride levels $(P<0.001)$, and a lower low-density lipoprotein cholesterol (LDL-C) $(P<0.001)$. These patients were also more likely to take less hydroxychloroquine $(P=0.044)$, more oral hypoglycemic agents $(P<0.001)$ and had a lower EQ-5D score $(P=0.025)$. In the multivariate analysis, when variables that belong to the composition of MetS were excluded, basal metabolic rate $(\mathrm{OR}=0.205,95 \% \mathrm{Cl}$ : $0.078-0.541$, $P=0.001)$ and Charlson comorbidity index score $(O R=2.191,95 \% \mathrm{Cl}$ : $1.280-3.751, P=0.004)$ remained significant predictors of MetS.

Conclusion: The development of MetS in RA patients was associated with basal metabolic rate and Charlson comorbidity index at baseline. Physicians should pay more attention to RA patients who have these risk factors to avoid CVD and cardiovascular mortality.

Disclosure of Interests: None declared

DOI: 10.1136/annrheumdis-2019-eular.3419

\section{THU0094 SERONEGATIVE RHEUMATOID ARTHRITIS: MILD NO MORE}

Sofia Pazmino ${ }^{1}$, Annelies Boonen ${ }^{2}$, Veerle Stouten ${ }^{1}$, Diederik De Cock ${ }^{1}$, Delphine Bertrand ${ }^{1,3}$, Kristien Van der Elst ${ }^{3}$, Johan Joly ${ }^{3}$, Rene Westhovens ${ }^{1,3}$ Patrick Verschueren ${ }^{1,3}$, CareRA study group. ${ }^{1}$ Katholieke Universiteit Leuven, Skeletal Biology and Engineering Research Centre, Leuven, Belgium; ${ }^{2}$ Maastrich University Medical Centre, Department of Internal Medicine, Division of Rheumatology, Maastricht, Netherlands; ${ }^{3}$ University Hospitals Leuven, Rheumatology, Leuven, Belgium

Background: Seronegative rheumatoid arthritis (RA) patients have been historically considered to have a milder disease. This confers a potential risk for under treatment. The quality of their response to early intensive therapies has not been thoroughly studied.

Objectives: To compare the disease course in seronegative and seropositive patients from the Care in early RA (CareRA) trial treated with COBRA Slim. 
Methods: The CareRA trial included 379 patients with early RA ( $\leq 1$ year) fulfilling 1987 or 2010 ACR criteria and naïve to disease modifying antirheumatic drugs (DMARDs). Patients were randomized to one of four different intensive treatment strategies based on methotrexate (MTX) monotherapy or synthetic (cs)DMARD combinations with or without glucocorticoid (GC) bridging. Before randomization, stratification into a high- or low-risk group according to classical poor prognostic markers (presence of erosions, positivity to rheumatoid factor (RF) and/or anti-citrullinated protein antibody (ACPA), and elevated disease activity) was conducted. One of these strategies, COBRA Slim, a combination of MTX and a prednisone remission induction scheme stepping-down from $30 \mathrm{mg}$ $\mathrm{QD}$, was initiated in 141 patients from both risk strata, of which 38 were negative for both RF and ACPA (seronegative). Following the treat-to-target principle, using the disease activity score in 28 joints with C-reactive protein (DAS28CRP), treatment adaptations were steered at low disease activity ( $\leq 3.2)$, while aiming for remission $(<2.6)$ as final outcome.

The "as observed" population was analysed for differences in disease activity and radiographic progression between seronegative and seropositive patients at several time points during the 2-year study using independent sample t-test, Man Whitney- $U$ or adjusted $X^{2}$ (Yate's correction for small samples). Survival analysis using Kaplan Meier was employed for time to first response to treatment. First response to treatment was defined as achieving first remission (DAS28CRP<2.6) or first relevant improvement $(\triangle \mathrm{DAS} 28 \mathrm{CRP}>1.2)$ after screening. Sensitivity analyses were applied on the complete CareRA cohort.

Results: Seronegative patients starting COBRA Slim $(n=38)$, had similar age (53 vs 51 years, $p=0.52$ ), body mass index ( 25.5 vs $26.7, p=0.08$ ), symptom duration ( 7.9 vs 7.2 months, $\mathrm{p}=0.23)$, presence of erosions (24\% vs $23 \%, p=0.99$ ) and gender distribution ( $82 \%$ vs $63 \%, p=0.06$ ), compared to seropositive patients $(n=103)$. However, parameters of disease activity were higher in seronegative patients at screening: DAS28CRP (5.1 vs $4.5, p=0.01), 28$ swollen joint count (8.2 vs 5.7 , $\mathrm{p}=0.02)$ and 28 tender joint count $(10.3$ vs $6.9, p=0.006)$. These results were corroborated when analysing the entire CareRA cohort.

The mean disease activity was significantly higher in seronegative patients at the first stages (week 8 and 16), but became comparable by year 1 and 2 (Table 1). There was a significant difference in time to first treatment response between seronegative and seropositive patients, despite being treated with the same strategy, as depicted in the inverted Kaplan Meier plot (Figure 1).

Conclusion: In conclusion, CareRA participants with seronegative RA had a higher initial disease activity, longer time to experience a first treatment response, but achieve comparable remission status as seropositive patients with COBRA Slim, a combination of MTX with moderate dose GC bridging. This provides further evidence that, seronegative RA can no longer be considered a milder form of disease and requires an equally intensive initial treat-to-target therapy as seropositive RA.

\section{Abstract THU0094 -Table 1. demographics}

\begin{tabular}{|c|c|c|c|}
\hline DAS28CRP at & SERONEGATIVE & SEROPOSITIVE & p-value \\
\hline W8 & $2.9 \pm 1.2(35)$ & $2.3 \pm 1.0(100)$ & 0.006 \\
\hline W16 & $2.6 \pm 1.1(35)$ & $2.2 \pm 0.9(100)$ & 0.023 \\
\hline Y1 & $2.3 \pm 1.0(33)$ & $2.3 \pm 1.0(93)$ & 0.929 \\
\hline Y2 & $2.0 \pm 0.9(27)$ & $2.1 \pm 0.8(87)$ & 0.162 \\
\hline SHARP VAN DER HEIJDE SCORE & SERONEGATIVE & SEROPOSITIVE & p-value \\
\hline wo & $1.3 \pm 2.2(33)$ & $1.1 \pm 2.2(78)$ & 0.156 \\
\hline W28 & $1.4 \pm 2.3(31)$ & $1.2 \pm 2.5(77)$ & 0.107 \\
\hline $\mathbf{Y}_{1}$ & $1.7 \pm 2.4(29)$ & $1.3 \pm 2.8(79)$ & 0.026 \\
\hline$Y_{2}$ & $1.5 \pm 2.2(22)$ & $1.8 \pm 3.4(70)$ & 0.384 \\
\hline$\Delta Y_{1}-w_{0}$ & $0.3 \pm 0.5(28)$ & $0.3 \pm 0.9(71)$ & 0.345 \\
\hline$\Delta$ Y2-w0 & $0.3 \pm 0.8(21)$ & $0.8 \pm 1.6(62)$ & 0.724 \\
\hline
\end{tabular}

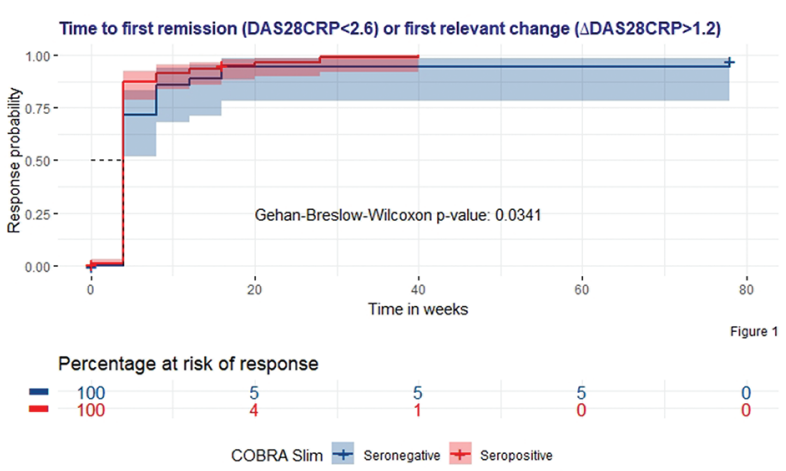

Abstract THU0094 - Figure 1

Disclosure of Interests: Sofia Pazmino: None declared, Annelies Boonen: None declared, Veerle Stouten: None declared, Diederik De Cock: None declared, Delphine Bertrand: None declared, Kristien Van der Elst: None declared, Johan Joly: None declared, Rene Westhovens Grant/research support from: Bristol-Myers Squibb, Consultant for: Celltrion, GalapagosGilead, Patrick Verschueren Grant/research support from: Unrestricted Pfizer Grant for Early RA research

DOI: 10.1136/annrheumdis-2019-eular.1940

\section{THU0095 CIGARETTE SMOKING AS PREDICTOR OF INADEQUATE RESPONSE TO METHOTREXATE IN RHEUMATOID ARTHRITISPATIENTS NAÏVE TO DMARDS}

Daniela Perra, Ignazio Cangemi, Mattia Congia, Marco Bartocci,

Elisabetta Chessa, Martina Dessì, Piero Mascia, Andrea Pau, Josephine Pilia, Ilaria Platè, Francesco Luca Renzullo, Alberto Floris, Matteo Piga, Alberto Cauli, Alessandro Mathieu. Università degli Studi e AOU di Cagliari, Scienze Mediche e Sanità Pubblica, Unità di Reumatologia, Cagliari, Italy

Background: Methotrexate (MTX) is the gold standard as first line treat ment in rheumatoid arthritis (RA). Identifying predictors of response to MTX should be crucial in the view of a personalized therapy.

Objectives: To identify prevalence and potential clinical baseline predictors of inadequate response (IR) to MTX in RA patients naïve to disease modifying anti-rheumatic drugs (DMARDs)*.

Methods: Data of 233 consecutive RA patients (according to ACR/EULAR 2010 criteria) naïve to traditional and biologic DMARDs, attending the AOU University Clinic, Rheumatology Unit, Monserrato (Cagliari), Italy, were analyzed. Table 1 shows baseline demographic, clinical and serological characteristics of the cohort. Patients failing to reach low disease activity state (DAS $28>3.2$ ) at 6 months (T1) since the beginning of MTX (T0) or patients undergoing therapeutic modification for persistently high disease activity (switch or addition of other traditional or biological DMARDs) before T1 were considered as IR. By univariate analysis, demographic, clinical and serological factors recorded at T0 were evaluated as potential predictors of IR at $T 1$. Afterwards, factors with $p<0.10$ were included in a logistic regression model, to identify independently associated factors to IR ( $p$ <.05). Odd-Ratio (OR) with $95 \%$ confidence interval $(\mathrm{Cl})$ was calculated.

Results: At T1, 104 patients (44.6\%) were classified as IR to MTX. In univariate analysis, factors significantly associated with IR were: female 